\title{
Erdős on polynomials
}

\author{
Vilmos Totik*
}

July 7, 2013

\begin{abstract}
Some results of Erdős on polynomials and some later developments are reviewed. The topics that this survey covers are: discrepancy estimates for zero distribution, orthogonal polynomials, distribution and spacing of their zeros and critical points of polynomials.
\end{abstract}

\section{Introduction}

Polynomials were Paul Erdős' favorite objects in analysis. He devoted many works to them, and in his problem lectures and papers he repeatedly returned to their theory. His major interest concerning them can be roughly divided into the following areas:

1) interpolation,

2) discrepancy theorems for zeros,

3) inequalities,

4) size and growth of polynomials,

5) geometric problems for lemniscates,

6) orthogonal polynomials,

7) spacing of zeros,

8) geometry of zeros of derivatives,

9) polynomials with integer coefficients.

He wrote most papers on interpolation. Several surveys have been devoted to Erdős' work on interpolation, see e.g. D. S. Lubinsky's and P. Vértesi's surveys [22] and [34] in the Erdős memorial volume and Vértesi's survey [35] in this volume. For inequalities, particularly for inequalities on the size of the derivatives of polynomials see T. Erdélyi's papers [5], [6]. We shall not touch

*Supported by European Research Council Advanced Grant No. 267055 
topic 4) (questions like how small the norm of a polynomial with \pm 1 coefficients on the unit circle can be, or if polynomials of degree at most $(1+\varepsilon) n$ interpolate in $n$ points, then does their minimal norm necessarily tend to infinity - for some interpolation data-when $\varepsilon \rightarrow 0$ ?) or topic 5) (questions like the minimal length of lemniscates or largest possible area for lemniscate domains) because there has not been a real breakthrough in those questions; see the papers [5] and [16]. Also, 9) (including questions on cyclotomic polynomials) has been adequately reviewed in [3].

Therefore, this survey will be devoted to some recent developments concerning

2) discrepancy theorems for zeros,

6) orthogonal polynomials,

7) spacing of zeros,

8) geometry of zeros of derivatives.

In the areas 2),6) and 7) most of Erdős' earlier papers were with Paul Turán, his lifelong friend. In their works in these directions interpolation has always been in the background. By now more powerful tools have been developed, but the impact of the Erdős-Turán papers has been enormous, and lasts until today.

\section{Discrepancy theorems}

We start with a problem of P. L. Chebyshev. In connection with a question in mechanics he was lead to replacing $x^{4}$ on $[-1,1]$ by a combination of smaller powers. He answered the general question: how well $x^{n}$ can be approximated by linear combination of smaller powers, i.e. he determined the quantity

$$
t_{n}=\inf _{P_{n}(x)=x^{n}+\cdots}\left\|P_{n}\right\|_{[-1,1]}
$$

where $\|\cdot\|_{K}$ denotes the supremum norm on a set $K$ :

$$
\left\|P_{n}\right\|_{K}=\sup _{z \in K}\left|P_{n}(z)\right|
$$

He found that

$$
t_{n}=\frac{2}{2^{n}}
$$

the extremal polynomials being the so called Chebyshev polynomials

$$
T_{n}(z)=\frac{1}{2^{n-1}} \cos (n \arccos x) .
$$

The Chebyshev polynomials have uniformly distributed zeros in the sense that if we project the zeros (all lying in $(-1,1)$ ) vertically onto the unit circle to get 


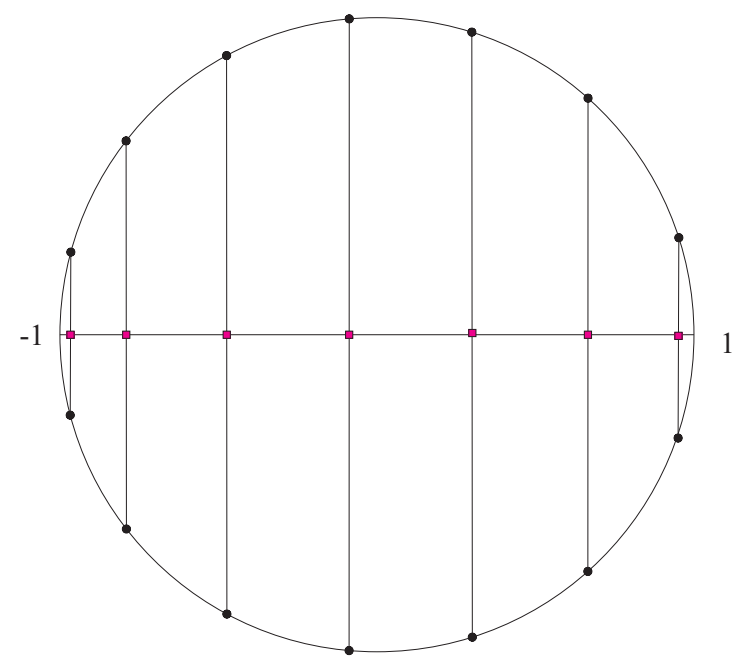

Figure 1: Uniform distribution of the Chebyshev zeros

$2 n$ points, then the points so obtained are uniformly distributed there in the sense that they divide the circle into $2 n$ equal arcs, see Figure 1.

What Erdős and Turán observed in [13] is that if the norm of a monic polynomial $P_{n}(z)=z^{n}+\cdots$ with all its zeros on $[-1,1]$ is not much larger than the minimal norm $t_{n}$, then the zeros of $P_{n}$ are almost like the zeros of the optimal polynomial $T_{n}$, i.e. in a sense the zeros (more precisely their projection on the unit circle) are uniformly distributed.

Theorem 2.1 (Erdős-Turán, 1940) If $P_{n}(z)=z^{n}+\cdots$ has all its zeros $\left\{x_{j}\right\}$ in $[-1,1]$ and

$$
\left\|P_{n}\right\|_{[-1,1]} \leq \frac{A_{n}}{2^{n}},
$$

then for any $-1 \leq a<b \leq 1$

$$
\left|\frac{\#\left\{x_{j} \in(a, b)\right\}}{n}-\frac{\arcsin b-\arcsin a}{\pi}\right| \leq \frac{8}{\log 3} \sqrt{\frac{\log A_{n}}{n}} .
$$

In particular, if

$$
\limsup _{n \rightarrow \infty}\left\|P_{n}\right\|_{[-1,1]}^{1 / n}=\frac{1}{2},
$$

then the distribution of the zeros is the arcsine distribution (note that, by Chebyshev's theorem, necessarily

$$
\left.\liminf _{n \rightarrow \infty}\left\|P_{n}\right\|_{[-1,1]}^{1 / n} \geq \frac{1}{2}\right) .
$$

In other words, the zeros of asymptotically minimal polynomials have arcsine distribution. 
Let us sketch the original argument of Erdős and Turán from [13]; a different approach will be given in the next section. First of all it is enough to prove the upper estimate

$$
\#\left\{x_{j} \in(a, b)\right\}-\frac{n(\arcsin b-\arcsin a)}{\pi} \leq \frac{4}{\log 3} \sqrt{n \log A_{n}},
$$

since the matching lower bound (with $4 / \log 3$ replaced by $8 / \log 3$ ) follows if we apply (3) to the two complementary intervals $[-1, a]$ and $[b, 1]$. Let $a=\cos \beta$, $b=\cos \alpha, \alpha, \beta \in[0, \pi]$, let $k=[n(\beta-\alpha) / \pi]$, and assume that there are at least $k+2 l$ zeros of $P_{n}$ in $[a, b]$. Consider the following modification of Chebyshev's problem: minimize the supremum norm of monic polynomials $Q_{n}(z)=z^{n}+\cdots$ on $[-1,1]$ under the constraint that the polynomial has $k+2 l$ zeros in $[a, b]$. There is an extremal polynomial $Q_{n}$, and by a simple variational argument $\left|Q_{n}\right|$ takes it maximal value (with respect to $[-1,1]$ ) in between any two of its consecutive zeros lying in $(a, b)$. According to a lemma of M. Riesz if a trigonometric polynomial of degree $n$ takes its maximum absolute value at a $\zeta$, then it has no zero in the interval $(\zeta-\pi / 2 n, \zeta+\pi / 2 n)$. Hence, the trigonometric polynomial $Q_{n}(\cos \theta)$ cannot have more than $[n(\beta-\alpha) / \pi]=k$ zeros in the interior of $(\alpha, \beta)$. Thus, to have $k+2 l$ zeros in $[\alpha, \beta]$ it must have $2 l$ zeros at $\alpha$ and $\beta$, so in at least one of the endpoints of $[\alpha, \beta]$ it has at least $l$ zeros. Therefore, by assumption,

$$
\frac{A_{n}}{2^{n}} \geq \min _{\psi_{n}}\left\|\psi_{n}\right\|_{[-1,1]},
$$

where $\psi_{n}$ is a polynomial which has a zero of multiplicity $l$ somewhere in $[-1,1]$. As a consequence,

$$
\frac{A_{n}}{2^{n}} \geq \min _{\psi_{n}}\left(\frac{1}{\pi} \int_{-1}^{1} \frac{\left|\psi_{n}(\xi)\right|^{2}}{\sqrt{1-\xi^{2}}} d \xi\right)^{1 / 2} .
$$

If $I_{n}\left(x_{0}\right)$ is the minimum value of the norm on the right for all $\psi_{n}$ which has a zero at $x_{0}$ of multiplicity $l$, then clearly

$$
\frac{A_{n}}{2^{n}} \geq \min _{x_{0} \in[-1,1]} I_{n}\left(x_{0}\right) .
$$

On applying the Zhoukowskii transformation $\zeta=\frac{1}{2}\left(z+\frac{1}{z}\right)$, it follows after multiplication by $z^{n}$ that $I_{n}\left(x_{0}\right)^{2}$ is the minimum of

$$
\frac{1}{\pi 2^{2 n+1}} \int_{-\pi}^{\pi}\left|\Psi_{2 n}\right|^{2}
$$

where the minimum is taken for all algebraic polynomials $\Psi_{2 n}=z^{2 n}+\cdots$ which have a zero of multiplicity $l$ at both $e^{ \pm i \theta_{0}}$, where $\cos \theta_{0}=x_{0}$. Reduce the 
assumption to have a single zero of multiplicity $l$, which then can be moved to any point on the unit circle by rotation, hence (by moving it to -1 )

$$
I_{n}\left(x_{0}\right)^{2} \geq \frac{1}{\pi 2^{2 n+1}} \min _{\Phi_{2 n-l}} \int_{|z|=1}\left|\Phi_{2 n-l}(z)\right|^{2}|1+z|^{2 l},
$$

the minimum being taken for all polynomials $\Phi_{2 n-l}(z)=z^{2 n-l}+\cdots$ of degree $2 n-l$.

Regard here $|1+z|^{2 l}$ as a weight function $w$ on the unit circle. It is well known (easily follows from orthogonality) that the minimum in (4) is attained for the $(2 n-l)$-th monic orthogonal polynomial with respect to $w$. Erdös and Turán figured out the explicit form

$$
l\left(\begin{array}{c}
2 n+l \\
l
\end{array}\right)(1+z)^{-2 l} \int_{-1}^{z}(z-t)^{l-1}(1+t)^{l} t^{2 n-l} d t
$$

for this orthogonal polynomial (once this form is given, one can rather easily check that it is a polynomial of degree $(2 n-l)$ with leading coefficient 1 and that it is orthogonal to every smaller power). In other words, the minimum in (4) is attained for this function, and the minimum value for the right-hand side in (4) can then be explicitly calculated and it is

$$
\frac{1}{2^{2 n}}\left(\begin{array}{c}
2 n+l \\
l
\end{array}\right)\left(\begin{array}{c}
2 n \\
l
\end{array}\right)^{-1} .
$$

Now Stirling's formula easily yields the lower bound

$$
\frac{1}{2^{n}} \exp \left[\left(\frac{\log 3}{4}\right)^{2} \frac{l^{2}}{n}\right]
$$

for $I_{n}\left(x_{0}\right)$. Thus,

$$
A_{n} \geq \exp \left[\left(\frac{\log 3}{4}\right)^{2} \frac{l^{2}}{n}\right],
$$

from which (3) immediately follows.

Indeed, this is a marvellous argument that gives a sharp estimate. However, it is also clear that it would be difficult to carry it over to Jordan curves or to disconnected sets. We shall see an alternative approach in the next section suitable in such situations.

\section{Some logarithmic potential theory}

Theorem 2.1 has been used in a number of situations, and has been extended to various directions. Erdős himself proved in [7] that if, besides (2) with $A_{n}=$ 
$O(1)$, the maximum of $\left|P_{n}\right|$ in between any two consecutive zeros is $\geq c / 2^{n}$, then

$$
\left|\frac{\#\left\{x_{j} \in(a, b)\right\}}{n}-\frac{\arcsin b-\arcsin a}{\pi}\right| \leq C \frac{\log n}{n} .
$$

To have a basis for generalization and to understand what is behind Theorem 2.1 (in particular, why the number $1 / 2$ and the arcsine distribution play such a prominent role) we need to consider what happens if the norm is taken on two intervals or on an even more general set. To do that we shall need to introduce some concepts from potential theory.

First of all, if $K$ is any compact set on the complex plane then we can form Chebyshev's problem on $K$ : what is the minimal norm $\left\|P_{n}\right\|_{K}$ of monic polynomials $P_{n}(z)=z^{n}+\cdots$ for a given $n$ ? Call this minimal norm $t_{n}(K)$. We assume that $K$ has infinitely many points (otherwise $t_{n}(K)=0$ for all large $n)$. It is immediate from the definition that $t_{n+m}(K) \leq t_{n}(k) t_{m}(K)$, i.e. $\log t_{n+m}(K) \leq \log t_{n}(K)+\log t_{m}(K)$, and then it is an easy exercise about sequences that the limit $\left(\log t_{n}(K)\right) / n$ exists (it is actually, equal to the infimum of all the numbers $\left.\left\{\left(\log t_{n}(K)\right) / n\right\}_{n=1}^{\infty}\right)$. In other words, the limit

$$
t(K)=\lim _{n \rightarrow \infty} t_{n}(K)^{1 / n}
$$

exists. This $t(K)$ is called the Chebyshev constant of $K$.

A related quantity is the so called logarithmic capacity that can be obtained via the equilibrium measure of $K$. If $\mu$ is a unit Borel-measure on $E$, then its logarithmic energy is

$$
I(\mu)=\iint \log \frac{1}{|z-t|} d \mu(z) d \mu(t) .
$$

If this is finite for some $\mu$, then there is a unique minimizing measure $\mu_{E}$, called the equilibrium measure of $E$. Examples:

- the equilibrium measure of $[-1,1]$ is

$$
d \mu_{[-1,1]}(t)=\frac{1}{\pi \sqrt{1-t^{2}}} d t,
$$

which is called the Chebyshev (or arcsine) distribution,

- if $C_{1}$ is the unit circle, then

$$
d \mu_{C_{1}}\left(e^{i t}\right)=\frac{1}{2 \pi} d t
$$

is the normalized arc measure on $C_{1}$.

Now with the minimal energy $I(K)=\inf _{\mu} I(\mu)$ the logarithmic capacity $\operatorname{cap}(K)$ of $K$ is defined as

$$
\operatorname{cap}(K)=e^{-I(K)} .
$$

Naturally, if all energies $I(\mu)$ are infinite (in which case there is no equilibrium measure), then $\operatorname{cap}(K)=0$.

Examples: 
- if $K$ is a disk/circle of radius $r$ then $\operatorname{cap}(K)=r$,

- $\operatorname{cap}([-1,1])=1 / 2$.

It is a simple fact (a consequence of the maximum principle for subharmonic functions) that if $P_{n}(z)=z^{n}+\cdots$, then

$$
\left\|P_{n}\right\|_{K} \geq \operatorname{cap}(K)^{n} .
$$

Now in the original Chebyshev problem and in Theorem 2.1 the constant $1 / 2$ appears because it is the logarithmic capacity of $[-1,1]$ : $\operatorname{cap}([-1,1])=1 / 2$. We can also see that Chebyshev's theorem $t_{n} \geq 2 \cdot(1 / 2)^{n}$ (see (1)) is just a sharper form of (7).

There is yet another related quantity introduced by M. Fekete, the transfinite diameter of $K$. For a given natural number $n$ we consider $n$ points on $K$ that maximize the product of their distances, i.e. for which the supremum

$$
\delta_{n}(K):=\sup _{z_{1}, \ldots, z_{n} \in K} \prod_{i \neq j}\left|z_{i}-z_{j}\right|
$$

is achieved. They may not be unique, the points in any maximizing system are called ( $n$-th) Fekete points on $K$. It is not difficult to show that the limit

$$
\delta(K)=\lim _{n \rightarrow \infty} \delta_{n}^{\frac{1}{n(n-1)}}(K)
$$

exists, and this limit is called the transfinite diameter of $K$.

It is a theorem due (different parts) to Fekete, A. Zygmund and G. Szegö (see e.g. [28, Theorem 5.5.2, Corollary 5.5.5]) that the three quantities: the Chebyshev constant (see (5)), the logarithmic capacity (see (6)) and the transfinite diameter (see (8)) are the same:

$$
\operatorname{cap}(K)=\delta(K)=t(K) .
$$

In modern mathematics mostly the logarithmic capacity is used. Of course, Erdős knew (9), but he never used logarithmic capacity - he was always talking about the transfinite diameter of a set (after all he must have heard it from Fekete himself).

After these preparations let us return to the Erdős-Turán discrepancy Theorem 2.1. It can be formulated as: for any $-1 \leq a<b \leq 1$

$$
\left|\frac{\#\left\{x_{j} \in(a, b)\right\}}{n}-\int_{a}^{b} \frac{1}{\pi \sqrt{1-x^{2}}} d x\right| \leq \frac{8}{\log 3} \sqrt{\frac{\log A_{n}}{n}} .
$$

Let $\delta_{x}$ the "Dirac delta" at $x$, i.e. the point mass 1 placed to $x$. If we introduce the normalized zero distribution

$$
\nu_{n}=\frac{1}{n} \sum_{j} \delta_{x_{j}}
$$



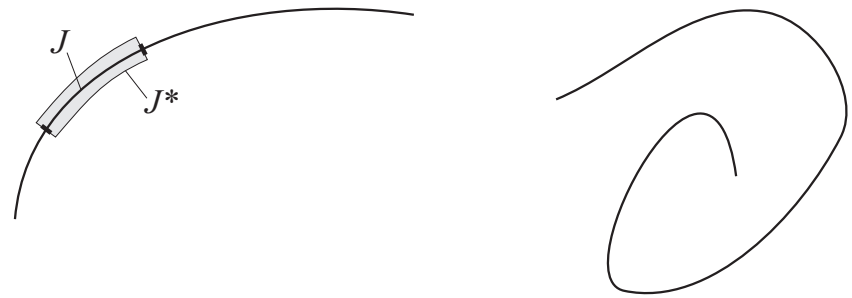

Figure 2: A neighborhood $J^{*}$ of a $J$ of $K$

associated with the zeros of $P_{n}$, then an equivalent form is: with the Chebyshev distribution

$$
d \mu_{[-1,1]}(x)=\frac{1}{\pi \sqrt{1-x^{2}}} d x
$$

for any interval $I \subset[-1,1]$

$$
\left|\nu_{n}(I)-\mu_{[-1,1]}(I)\right| \leq \frac{8}{\log 3} \sqrt{\frac{\log A_{n}}{n}} .
$$

Note that here $\mu_{[-1,1]}$ is the equilibrium measure of $[-1,1]$, and this is the appropriate form for generalizations.

Let $K$ be a finite union of smooth Jordan arcs (homeomorphic images of $[0,1])$, and let $P_{n}(z)=z^{n}+\cdots$ be a monic polynomial. Recall from (7) that we necessarily have $\left\|P_{n}\right\|_{K} \geq \operatorname{cap}(K)^{n}$, so asymptotically minimal polynomials on $K$ satisfy

$$
\lim _{n \rightarrow \infty}\left\|P_{n}\right\|_{K}^{1 / n}=\operatorname{cap}(K)
$$

Erdős and Turán repeatedly mentioned (see e.g. [12, p. 165]) a theorem of Fekete that was communicated to them verbally which claimed that if all zeros of $P_{n}$ are on single Jordan curve $K$ then (11) is true if and only if the zeros are distributed uniformly with respect to the conformal map $\Phi$ of $\mathbf{C} \backslash K$ onto the exterior of the unit disk (i.e. the $\Phi$-image of the zeros is uniformly distributed on the unit circle). This seems to be the first extension of the Erdös-Turán discrepancy theorem from an interval to a general curve. Note that the equilibrium measure $\mu_{K}$ is the $\Phi$-pull-back of the normalized arc-measure on the unit circle: $\mu_{K}(E)=|\Phi(E)| / 2 \pi$, so Fekete's theorem can be rephrased saying that (11) is true if and only if the asymptotic distribution of the zeros is the equilibrium distribution.

The most general form of the Erdős-Turán discrepancy theorem is due to V. V. Andrievskii and H-P. Blatt [1, Theorem 2.4.2]. It involves the quantity $A_{n}$ for which

$$
\left\|P_{n}\right\|_{K} \leq A_{n} \operatorname{cap}(K)^{n},
$$

and neighborhoods $J^{*}$ of subarcs $J \subset K$ depicted in Figure 2. 
Theorem 3.1 (Andrievskii-Blatt, 1995-2000) Let $K$ be a finite union of disjoint smooth Jordan arcs; let $\nu_{n}$ be the normalized zero distribution of a monic polynomial $P_{n}$ of degree $n$ and let $A_{n}$ be defined by (12). Then for any subarc $J \subset K$ we have

$$
\left|\nu_{n}\left(J^{*}\right)-\mu_{K}\left(J^{*}\right)\right| \leq C \sqrt{\frac{\log A_{n}}{n}},
$$

where $C$ depends only on $K$.

In particular, if $\left\|P_{n}\right\|_{K}^{1 / n} \rightarrow \operatorname{cap}(K)$, then $\nu_{n} \rightarrow \mu_{K}$, as was claimed by Fekete for one arc.

When $K$ consists of piecewise smooth arcs, then the square root on the right of (13) must be replaced by a different power that depends on the angles in between the different smooth $\operatorname{arcs}$ of $K$.

To give a flavor of a potential-theoretic argument, in closing this section we give a "modern" approach to the discrepancy Theorem 2.1 of Erdős and Turán (modulo the exact constant).

Let $\mu=\mu_{[-1,1]}$ be the equilibrium measure of $[-1,1]$ (the arcsine measure). It is again enough to prove an appropriate upper bound for $\left(\nu_{n}-\mu\right)([-1, a])$, $a \in(-1,1)$. For simplicity assume that $a \in[-2 / 3,2 / 3]$. For a $\delta>0$ consider the pair of intervals $I^{+}:=[-1, a], I^{-}:=[a+\delta, 1]$ (a so called condenser). All the constants $c_{i}$ below depend on $\delta$, but the important $c_{2}, c_{3}, c_{5}, c_{6}$ and $c_{7}$ lie in between two fixed constants independent of $\delta$. The following are rather simple facts from potential theory. There is a signed measure $\sigma=\sigma^{+}-\sigma^{-}$(the so called condenser equilibrium measure) such that $\sigma^{ \pm}$are positive probability measures, $\sigma^{ \pm}$is supported on $I^{ \pm}$, the logarithmic potential

$$
U^{\sigma}(z)=\int \log \frac{1}{|z-t|} d \sigma(t)
$$

of $\sigma$ equals a constant $c_{1}$ on $I^{-}$and another constant $c_{1}+c_{2} / \log (1 / \delta)$ on $I^{+}$, and everywhere else it lies in between these two constants. It is also true that if $I=I^{+} \cup I^{-}$, then the equilibrium measure $\mu_{I}$ of $I$ majorizes $\sigma^{+}+\sigma^{-}$: $\sigma^{+}+\sigma^{-} \leq\left(c_{3} / \delta \log (1 / \delta)\right) \mu_{I}$, furthermore (by Frostman's theorem [28, Theorem 3.3.4]) the equilibrium potential $U^{\mu_{I}}$ is constant $c_{4}(=\log 1 / \operatorname{cap}(I))$ on $I$, it is everywhere else less than $c_{4}$, but on the interval $[a, a+\delta]$ it is bigger than $c_{4}-c_{5} \delta$.

Using these, we obtain from Fubini's theorem

$$
-\int U^{\sigma} d\left(\mu-\nu_{n}\right)=-\int U^{\mu-\nu_{n}} d \sigma .
$$

Here, since by Frostman's theorem [28, Theorem 3.3.4] the equilibrium potential $U^{\mu}$ is identically equal to $\log 1 / \operatorname{cap}([-1,1])=\log 2$ on $[-1,1]$, we have

$$
U^{\mu-\nu_{n}}(z)=\log 2+\log \left|P_{n}(z)\right|^{1 / n} \leq \frac{\log A_{n}}{n}, \quad z \in[-1,1],
$$


by the definition of the constant $A_{n}$ in (2). Hence, since $\sigma(\mathbf{C})=0$, we can continue the preceding line as

$-\int U^{\mu-\nu_{n}} d \sigma=\int\left(\frac{\log A_{n}}{n}-U^{\mu-\nu_{n}}\right) d \sigma \leq \int\left(\frac{\log A_{n}}{n}-U^{\mu-\nu_{n}}\right) d\left(\sigma^{+}+\sigma^{-}\right)$.

Replace on the right $\sigma^{+}+\sigma^{-}$by the larger $\left(c_{3} / \delta \log (1 / \delta)\right) \mu_{I}$, and apply again Fubini's theorem to conclude the following bound for the right-hand side

$$
\frac{c_{3}}{\delta \log (1 / \delta)} \frac{\log A_{n}}{n}-\frac{c_{3}}{\delta \log (1 / \delta)} \int U^{\mu_{I}} d\left(\mu-\nu_{n}\right) .
$$

In the last integral $U^{\mu_{I}}$ can be replaced by $U^{\mu_{I}}-c_{4}$ (the total mass of $\mu-\nu_{n}$ is 0 ), and since $U^{\mu_{I}}-c_{4}=0$ on $I$, the integral becomes

$$
\int_{a}^{a+\delta}\left(U^{\mu_{I}}-c_{4}\right) d\left(\mu-\nu_{n}\right)
$$

Since $U^{\mu_{I}}-c_{4} \leq 0$, if we omit here the measure $-\nu_{n}$ then we decrease the integral. Finally, from $\mu([a, a+\delta]) \leq c_{6} \delta$ and from $U^{\mu_{I}}-c_{4} \geq-c_{5} \delta$ on $[a, a+\delta]$ we can conclude

$$
-\int U^{\sigma} d\left(\mu-\nu_{n}\right) \leq \frac{c_{3}}{\delta \log (1 / \delta)} \frac{\log A_{n}}{n}+\frac{c_{3}}{\delta \log (1 / \delta)} c_{5} c_{6} \delta^{2} .
$$

On the left we can replace $U^{\sigma}$ by $U^{\sigma}-c_{1}$, and then the left-hand side becomes

$$
-\frac{c_{2}}{\log (1 / \delta)}\left(\mu-\nu_{n}\right)([-1, a])-\int_{a}^{a+\delta}\left(U^{\sigma}-c_{1}\right) d\left(\mu-\nu_{n}\right) .
$$

Since the last signed integral is at least

$$
-\int_{a}^{a+\delta}\left(U^{\sigma}-c_{1}\right) d \mu \geq-\frac{c_{2}}{\log (1 / \delta)} \mu([a, a+\delta]) \geq-\frac{c_{2} c_{6} \delta}{\log (1 / \delta)},
$$

we finally obtain

$$
-\frac{c_{2}}{\log (1 / \delta)}\left(\mu-\nu_{n}\right)([-1, a])-\frac{c_{2} c_{6} \delta}{\log (1 / \delta)} \leq \frac{c_{3}}{\delta \log (1 / \delta)} \frac{\log A_{n}}{n}+c_{3} c_{5} c_{6} \frac{\delta}{\log (1 / \delta)},
$$

i.e.

$$
\left(\nu_{n}-\mu\right)([-1, a]) \leq c_{7}\left(\frac{\log A_{n}}{n \delta}+\delta\right) .
$$

Now the $\delta=\sqrt{\frac{\log A_{n}}{n}}$ choice gives the desired

$$
\left(\nu_{n}-\mu\right)([-1, a]) \leq 2 c_{7} \sqrt{\frac{\log A_{n}}{n}} .
$$

It is clear from this proof that with appropriate modifications it can be given on smooth Jordan curves, or even on unions of such curves. 


\section{A second discrepancy theorem}

Erdős and Turán had a second discrepancy theorem for the zeros of polynomials which had equally important consequences.

Note first of all, that the results from the preceding sections have no direct analogues for Jordan curves (homeomorphic images of the unit circle). Consider e.g. the polynomials $z^{n}$ on the unit circle $C_{1}$. These have norm 1 , which is the ( $n$-th power of the) capacity of $C_{1}$, and still all their zeros lie far from $C_{1}$, which carries the equilibrium distribution. In this section we shall discuss how to get discrepancy theorems for the zeros on Jordan curves.

Let us start with a theorem of $\mathrm{R}$. Jentzsch from 1918: if the radius of convergence of a power series $\sum_{j=0}^{\infty} a_{j} z^{j}$ is 1 , then the zeros of (all) the partial sums $\sum_{0}^{n} a_{j} z^{j}, n=1,2, \ldots$ are dense at ever point of the unit circle. Szego" made a refinement in 1922: there is a sequence $n_{1}<n_{2}<\cdots$ such that if $z_{j, n}=$ $r_{j, n} e^{i \theta_{j, n}}, 1 \leq j \leq n$ are the zeros of $\sum_{0}^{n} a_{j} z^{j}$, then $\left\{\theta_{j, n_{k}}\right\}_{1}^{n_{k}}$ is asymptotically uniformly distributed (and $r_{j, n_{k}} \approx 1$ for most $j$, i.e. for every $\varepsilon>0$ there are only $o\left(n_{k}\right)$ zeros outside the ring $\left.1-\varepsilon<|z|<1+\varepsilon\right)$.

In connection with these Erdős and Turán proved in [15] the following. Let $P_{n}(z)=a_{n} z^{n}+\cdots+a_{0}$ be a polynomial with zeros $z_{j}=r_{j} e^{i \theta_{j}}, 1 \leq j \leq n$, and let $C_{1}=\{|z|=1\}$ be the unit circle.

Theorem 4.1 (Erdős-Turán, 1950) For any interval $J \subset[-\pi, \pi]$

$$
\left|\frac{\#\left\{\theta_{j} \in J\right\}}{n}-\frac{|J|}{2 \pi}\right| \leq 16 \sqrt{\frac{\log \left(\left\|P_{n}\right\|_{C_{1}} / \sqrt{\left|a_{0} a_{n}\right|}\right)}{n}} .
$$

Note that

$$
\left\|P_{n}\right\|_{C_{1}} \leq \sum_{j}\left|a_{j}\right|
$$

so we can replace $\left\|P_{n}\right\|_{C_{1}}$ on the right of (14) by $\sum_{j}\left|a_{j}\right|$ :

$$
\left|\frac{\#\left\{\theta_{j} \in J\right\}}{n}-\frac{|J|}{2 \pi}\right| \leq 16 \sqrt{\frac{\log \left(\sum_{j}\left|a_{j}\right| / \sqrt{\left|a_{0} a_{n}\right|}\right)}{n}} .
$$

An immediate consequence is that $P_{n}$ has at most

$$
32 \sqrt{n \log \left(\sum_{j}\left|a_{j}\right| / \sqrt{\left|a_{0} a_{n}\right|}\right)}
$$

real zeros (jus apply the inequality (15) to the degenerate intervals $J=\{0\}$ and $J=\{\pi\}$ ). This is better than previous estimates of B. Bloch, G. Pólya and E. Schmidt on the number of real zeros of polynomials, and recaptures a theorem (modulo a constant) of I. Schur. 


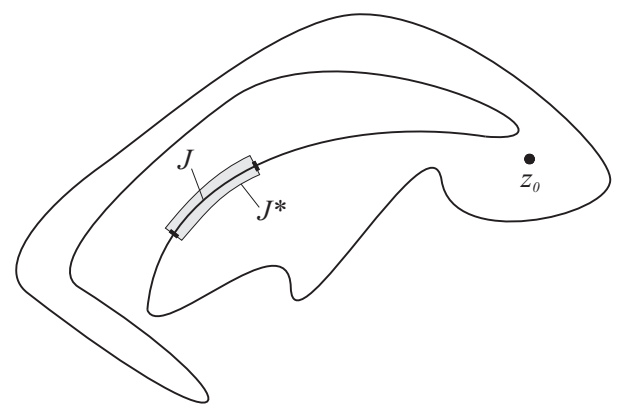

Figure 3: The position of $z_{0}$ and the neighborhood $J^{*}$ of an $\operatorname{arc} J$

Next, consider Szegő's theorem mentioned before. In considering $\sum_{j=0}^{\infty} a_{j} z^{j}$ we may assume $a_{0} \neq 0$. Now the radius of convergence of this power series is 1 precisely if

$$
\limsup \left|a_{n}\right|^{1 / n}=1,
$$

and this easily implies the existence of a subsequence $\left\{n_{k}\right\}$ with

$$
C_{n_{k}}:=\left(\frac{\sum_{j=0}^{n_{k}}\left|a_{j}\right|}{\sqrt{\left|a_{0} a_{n}\right|}}\right)^{1 / n_{k}} \rightarrow 1 .
$$

If $z_{j, n}=r_{j, n} e^{i \theta_{j}, n}$ are the zeros of $\sum_{j=0}^{n} a_{j} z^{j}$, then, by (15), we have

$$
\left|\frac{\#\left\{\theta_{j, n_{k}} \in J\right\}}{n_{k}}-\frac{|J|}{2 \pi}\right| \leq 16 \sqrt{\log C_{n_{k}}} \rightarrow 0,
$$

which shows the uniform distribution of the arguments of the zeros. A relatively simple argument gives that the number of zeros outside any ring $1-\varepsilon<|z|<$ $1+\varepsilon$ tends to zero. Thus, one can easily get both the Jentzsch and the Szegö theorem mentioned before from the Erdős-Turán inequality (14).

This second discrepancy theorem of Erdős and Turán has also been extended in various directions, see e.g. [2], [17]. We only mention the following generalization due to Andrievskii and Blatt [1, Theorem 2.4.5]. Note first of all that if $a_{n}=1$, then $\sqrt{\left|a_{0} a_{n}\right|}$ in (14)-(15) is just $\sqrt{\left|P_{n}(0)\right|}$, so the following statement is a direct generalization.

Theorem 4.2 (Andrievskii-Blatt, 1995-2000) If $\Gamma$ is a smooth Jordan curve, $z_{0}$ a fixed point inside $\Gamma, P_{n}(z)=z^{n}+\cdots$ and

$$
B_{n}:=\frac{\left\|P_{n}\right\|_{\Gamma}}{\sqrt{\operatorname{cap}(\Gamma)^{n}\left|P_{n}\left(z_{0}\right)\right|}},
$$

then for all arc $J \subset \Gamma$

$$
\left|\frac{\#\left\{z_{j} \in J^{*}\right\}}{n}-\mu_{\Gamma}(|J|)\right| \leq C_{0} \sqrt{\frac{\log B_{n}}{n}} .
$$


See Figure 3 for the position of $z_{0}$ and $J^{*}$. Recall that $\mu_{\Gamma}$ is the equilibrium measure of $\Gamma$.

In some form the theorem is actually true for a family of Jordan curves.

The Erdős-Turán discrepancy theorems have motivated many later works; eventually a deep theory of discrepancy of signed measures have evolved, see e.g. the book [1].

\section{Orthogonal polynomials on a finite interval}

Let $\rho$ be a positive Borel-measure with compact support on the complex plane. The orthonormal polynomials $p_{n}(z)=\gamma_{n} z^{n}+\cdots, n=0,1, \ldots$, with respect to $\rho$ are the unique polynomials with $\gamma_{n}>0$ and

$$
\int p_{n} \overline{p_{m}} d \rho= \begin{cases}0 & \text { if } n \neq m \\ 1 & \text { if } n=m\end{cases}
$$

If $S$ is the support of $\rho$, then for the leading coefficients $\gamma_{n}$ it is always true (see $[30$, Corollary 1.1.7]) that

$$
\frac{1}{\operatorname{cap}(S)} \leq \liminf _{n \rightarrow \infty} \gamma_{n}^{1 / n} .
$$

Earlier results on orthogonal polynomials had mostly been about the classical Hermite, Jacobi and Laguerre polynomials. Erdős and Turán were among the first (along with T. J. Stieltjes, S. N. Bernstein and Szegö) who got general results for rather general measures. However, they were always considering the case when the support is $[-1,1]$.

Theorem 5.1 (Erdös-Turán, 1940) If the support of $\rho$ is $[-1,1], d \rho(x)=$ $w(x) d x$ and $w>0$ almost everywhere, then

(a) the asymptotic zero distribution of $p_{n}$ is the Chebyshev distribution,

(b)

$$
\left|p_{n}(z)\right|^{1 / n} \rightarrow\left|z+\sqrt{z^{2}-1}\right|, \quad z \notin[-1,1] .
$$

In the latter limit the convergence is uniform on compact subsects of $\overline{\mathbf{C}} \backslash[-1,1]$. In particular, it also follows from this theorem that

$$
\lim _{n \rightarrow \infty} \gamma_{n}^{1 / n}=2
$$

(c.f. $(16)$ and note that $\operatorname{cap}([-1,1])=1 / 2)$.

Since the classical Jacobi polynomials have also this behavior, one could say that the condition " $w>0$ almost everywhere on $[-1,1]$ " implies classical 
behavior. This innocently looking condition turns out to be quite crucial, e.g. we shall see that the behavior of $p_{n}$ and their zeros is totally different if $\rho$ vanishes on a subinterval of $[-1,1]$.

It took about 40 years for sharper results, when, in 1977-82, E. A. Rakhmanov $[26]-[27]$ proved that not just (b) is true, but also the stronger

$$
\frac{p_{n+1}(z)}{p_{n}(z)} \rightarrow z+\sqrt{z^{2}-1}, \quad z \notin[-1,1] .
$$

H. Widom showed in 1967 that no ratio asymptotics as in (17) is possible if the support is not connected. Thus, in that case one should settle with an analogue of (b) in Theorem 5.1. To state this analogue we need the concept of Green's function. Let $\Omega$ be the unbounded connected component of $\overline{\mathbf{C}} \backslash S$ (where $S$ is the support of $\rho$ ), and we assume that $S$ has positive logarithmic capacity, so it has equilibrium measure $\mu_{S}$ (see Section 3 ). With this equilibrium measure the Green's function $g_{\overline{\mathbf{C}} \backslash S}(z)$ of $\overline{\mathbf{C}} \backslash S$ with pole at infinity is the function

$$
g_{\overline{\mathbf{C} \backslash S}}(z)=\int \log |z-t| d \mu_{S}(t)-\log \operatorname{cap}(K), \quad z \in \Omega
$$

(it is customary to set $g_{\overline{\mathbf{C} \backslash S}}$ to be zero outside $\Omega$ ). An alternative definition is that $g_{\overline{\mathbf{C} \backslash S}}$ is the unique nonnegative harmonic function in $\Omega$ which behaves at infinity as $\log |z|+$ const and at "almost all points" of $\partial \Omega$ ("almost all" with respect to logarithmic capacity) has zero limit. We also assume that there is no set of zero capacity that carries the measure $\rho$.

Examples:

- if $C_{R}$ is the circle about the origin of radius $R$, then

$$
g_{\overline{\mathbf{C}} \backslash C_{R}}(z)=\log (|z| / R),
$$

$$
g_{\overline{\mathbf{C} \backslash[-1,1]}}(z)=\log \left|z+\sqrt{z^{2}-1}\right|
$$

Thus, the function $\left|z+\sqrt{z^{2}-1}\right|$ appearing in (b) in Theorem 5.1 can be recognized as the exponential of the Green's function of $\overline{\mathbf{C}} \backslash[-1,1]$, while the Chebyshev distribution in part (a) is the equilibrium distribution. These guide us to a general formulation.

In discussing the general form of Theorem 5.1 for simplicity assume that $S=\operatorname{supp}(\mu)$ has connected complement and empty interior (e.g. $S \subset \mathbf{R}$ ), and $S$ is regular in the sense that $g_{\overline{\mathbf{C}} \backslash S}(z) \rightarrow 0$ as $z \rightarrow \zeta \in \partial \Omega, z \in \Omega$, for all $\zeta \in \partial \Omega$. This latter condition is a mild one, most sets that naturally appear satisfy it.

The next result has evolved through the works of J. Ullman, Erdős, Turán, Widom, H. Stahl and W. Van Assche; in the presented form it is taken from the monograph [30].

Theorem 5.2 The following are pairwise equivalent. 
(i) The asymptotic zero distribution of the orthogonal polynomials $p_{n}$ is the equilibrium distribution $\mu_{S}$ of the support $S$ of $\rho$,

(ii)

$$
\gamma_{n}^{1 / n} \rightarrow \frac{1}{\operatorname{cap}(S)} \quad \text { as } n \rightarrow \infty,
$$

(iii)

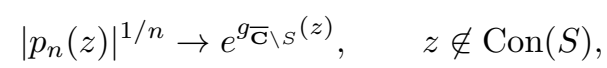

(iv) for all (or one) $0<q<\infty$

$$
\sup _{P_{n}} \frac{\left\|P_{n}\right\|_{S}^{1 / n}}{\left\|P_{n}\right\|_{L^{q}(\rho)}^{1 / n}} \rightarrow 1 .
$$

If either of these properties holds then we say that $\rho$ belongs to the $\mathbf{R e g}$ class. $\rho \in \mathbf{R e g}$ is a very weak regularity assumption on the measure. $\rho \in \mathbf{R e g}$, i.e. regular behavior means roughly that the measure is not too thin on any part of its support, and in terms of the orthogonal polynomials it means that the orthogonal polynomials behave non-pathologically.

Condition (ii) claims that the leading coefficients are asymptotically minimal (see (16)), while property (iv) says that in $n$-th root sense the integral norms of polynomials with respect to $\rho$ are about the same (of the same order) as their supremum norm on the support $S$ of $\rho$ (note that $\left\|P_{n}\right\|_{L^{q}(\mu)} \leq \mu(\mathbf{C})^{1 / q}\left\|P_{n}\right\|_{S}$ ).

If $S$ has nonzero interior or $\overline{\mathbf{C}} \backslash S$ is not connected, then the equivalence of (ii)-(iv) is still true; but the asymptotic zero distribution may not be $\mu_{S}$. Consider e.g. the arc-measure on the unit circle or the area-measure on the unit disk as $\rho$. In these cases the $n$-th orthonormal polynomial is a constant multiple of $z^{n}$, which has all its zeros at the origin, while the equilibrium measure is the normalized arc-measure on the unit circle.

With the Reg class we can see that Theorem 5.1 claims nothing else than $S=[-1,1]$ and $d \rho(x)=w(x) d x$ with $w>0$ almost everywhere on $[-1,1]$ imply $\rho \in$ Reg. The condition " $w>0$ almost everywhere" is called the (original) Erdős-Turán criterion. In the monograph [30] we called

$$
\frac{d \rho(z)}{d \mu_{S}}>0 \quad \mu_{S}-\text { almost everywhere }
$$

the general Erdös-Turán criterion. On the left the derivative is the RadonNikodym derivative of $\rho$ with respect to the equilibrium measure $\mu_{S}$ of $S=$ $\operatorname{supp}(\rho)$. (When $S=[-1,1]$ then we have $d \mu_{S}(x)=\left(\pi \sqrt{1-x^{2}}\right)^{-1} d x$ and then clearly (18) is true if and only if

$$
\frac{d \rho(x)}{d x}>0 \quad \text { almost everywhere on }[-1,1],
$$

so (18) is, indeed, a generalization of the original Erdős-Turán criterion.) In the general case we have (see [30, Theorem 4.1.1]) 
Theorem 5.3 (Stahl-Totik, 1990) The Erdös-Turán criterion (18) implies $\rho \in$ Reg.

By now there are many weaker (more powerful) criteria for regularity, see [30, Ch. 4], but no necessary and sufficient condition is known. The only necessary condition (in terms of the size of the measure $\rho$ ) is the following: if the support of $\rho$ is $[-1,1]$ and $\rho \in \mathbf{R e g}$, then for all $\eta>0$ the capacity of the set

$$
E_{\eta, n}:=\left\{x \in[-1,1] \mid \rho\left(\left[x-\frac{1}{n}, x+\frac{1}{n}\right]\right)>e^{-\eta n}\right\}
$$

tends to $1 / 2$ (the capacity of $[-1,1]$ ) as $n \rightarrow \infty$.

A closest sufficient condition is

Criterion $\lambda^{*}$ : the support of $\rho$ is $[-1,1]$ and for every $\eta>0$ the measure of the set $E_{\eta, n}$ tends to $2($ as $n \rightarrow \infty)$.

Thus, criterion $\lambda^{*}$ implies $\rho \in \mathbf{R e g}$. An analogous criterion for general sets using capacity is

Criterion $\Lambda^{*}$ : there is an $L$ such that the capacity of the set

$$
\left\{z \in S \mid \rho\left(\Delta_{1 / n}(z)\right)>n^{-L}\right\}
$$

tends, as $n \rightarrow \infty$, to the capacity $\operatorname{cap}(S)$ of the support $S$ of $\rho$ (here $\Delta_{1 / n}(z)$ denotes the disk of radius $1 / n$ with center at $z$ ).

In [12] Erdős claimed to had proven a necessary and sufficient condition for $\rho \in \mathbf{R e g}$, but he did not state the condition and he had never published it. He periodically returned to the following statement conjectured by him which, according to [8], he had never been able to fully prove: if $S=[-1,1]$ and $d \rho(x)=w(x) d x$ with a bounded $w$, then $\rho \in \mathbf{R e g}$ if and only if $\operatorname{cap}\left(E_{\varepsilon}\right) \rightarrow 1 / 2$ as $\varepsilon \rightarrow 0$, where $E_{\varepsilon}$ is any set obtained from $\{x \mid w(x)>0\}$ by removing a subset of measure $<\varepsilon$.

This seems to be still open, though the sufficiency easily follows from Criterion $\Lambda^{*}$ in (19).

Regularity plays an important role in the general theory of orthogonal polynomials. It gives a weak global condition under which many properties of orthogonal polynomials can be localized. We shall see examples in the next section.

\section{Spacing of zeros of orthogonal polynomials}

Let again $d \rho(x)=w(x) d x$ be a measure on $[-1,1], p_{n}$ the orthonormal polynomials with respect to $\rho$ and let $x_{j}=x_{j, n}=\cos \theta_{j, n}=\cos \theta_{j}, \theta_{j} \in[0, \pi]$, be the zeros of $p_{n}$ in increasing order. In this case all zeros lie in $(-1,1)$, and in the 1930's and 1940's Erdős and Turán had many results on the spacing of these zeros. For the following discussion we speak of rough spacing when

$$
\theta_{j-1}-\theta_{j} \sim \frac{1}{n}, \quad \text { i.e. } \frac{c_{1}}{n} \leq \theta_{j-1}-\theta_{j} \leq \frac{c_{2}}{n} .
$$


Fine zero spacing means

$$
\theta_{j-1}-\theta_{j} \approx \frac{\pi}{n}, \quad \text { i.e. } \quad n\left(\theta_{j-1}-\theta_{j}\right) \rightarrow \frac{\pi}{n} .
$$

For example, classical (Jacobi) polynomials obey fine spacing inside $(-1,1)$.

As a first result on rough spacing we mention [12, Theorem VIII] which was the first general result on local rough spacing.

Theorem 6.1 (Erdös-Turán, 1940) If the support of $\rho$ is $[-1,1], d \rho(x)=$ $w(x) d x$ with a $w$ that lies in between two positive constants on some interval $[a, b]$, then inside any interval $[a+\varepsilon, b-\varepsilon]$ the zeros of the orthogonal polynomials obey rough spacing.

By now it has become clear that rough spacing of zeros is basically equivalent to $\rho$ being a doubling measure:

$$
\rho(2 I) \leq C \rho(I) \quad \text { for all intervals } I \subset[-1,1] .
$$

Here $2 I$ is the interval $I$ enlarged twice from its center. More precisely, the following was proved in [24, Theorem 1].

Theorem 6.2 (Mastroianni-Totik, 2010) If $\rho$ is doubling on $[-1,1]$, then $p_{n}$ obey rough zero spacing (on the whole interval $[-1,1]$ ).

This includes all previous result on rough spacing of zeros. Furthermore, if $\rho$ is doubling then for the so called Cotes numbers

$$
\frac{1}{\lambda_{n, j}}=\sum_{k=0}^{n} p_{k}\left(z_{n, j}\right)^{2}
$$

(these appear in Gaussian quadrature) we have

$$
0<c \leq \frac{\lambda_{n, j+1}}{\lambda_{n, j}} \leq C
$$

uniformly in $n$ and $j$. Now this uniform boundedness and rough zero spacing is actually equivalent to the doubling condition, see [24, Theorem 3]. It is an open problem if rough spacing alone is equivalent to $\rho$ being doubling (in other words, if rough spacing (20) implies (22)).

These results also have a local version, see [32] and [33].

Fine zero spacing requires more smoothness on the weight. It follows from some deep results of Szegö and Bernstein that if $w \geq c>0$ (with $d \rho(x)=$ $w(x) d x)$ on $[-1,1]$ and $w$ is twice differentiable on an interval, then inside this interval there is a strong asymptotic formula for the orthogonal polynomials which easily implies fine zero spacing. Erdős and Turán found this approach too restrictive (too "big gun" is used), and they gave the following beautiful theorem. 
Theorem 6.3 (Erdös-Turán, 1940) If $d \rho(x)=w(x) d x$ where $w>0$ is continuous on $[-1,1]$, then $p_{n}$ obeys fine zero spacing for the zeros lying in any subinterval $(-1+\varepsilon, 1-\varepsilon)$, i.e.

$$
\theta_{j-1}-\theta_{j} \approx \frac{\pi}{n}
$$

there.

This is no longer true if $w$ is allowed to vanish somewhere on $[-1,1]$, and it is a delicate question what properties of $w$ imply fine zero spacing. It has turned out that this question is related to some universality problems in random matrix theory, namely to a well defined and "universal" (i.e. independent of $\rho$ ) behavior of the kernel function

$$
\sum_{k=0}^{n} p_{k}(z+a / n) p_{k}(z+b / n) \quad a, b \in \mathbf{C} .
$$

D. S. Lubinsky [21] proved in 2009 this universality under the $\rho \in \mathbf{R e g}$ global condition and under local continuity and positivity of $w$. The following is a consequence from [19]:

Theorem 6.4 (Levin-Lubinsky, 2008) If $\rho \in \mathbf{R e g}$ and $w$ is continuous and positive at $z_{0} \in(-1,1)$, then (23) is true for the zeros $x_{j}$ that lie close to $x_{0}$ : $x_{j}-z_{0}=O(1 / n)$.

Now what happens if $\rho$ vanishes on some subinterval of $[-1,1]$, or more generally, if $d \rho(x)=w(x) d x$ is supported on some general compact set $S$ of the real line? Then the equilibrium measure $\mu_{S}$ of $S$ enters into zero spacing. More precisely, we need the density of that equilibrium measure: if $I \subset S$ is an interval, then $\mu_{S}$ is absolutely continuous on $I$ with respect to Lebesguemeasure: $d \mu_{S}(t)=\omega_{S}(t) d t$, and its density $\omega_{S}$ is a $C^{\infty}$ function there.

Examples:

- for the unit circle/disk the equilibrium density is the identically $1 / 2 \pi$ function on the unit circle,

$$
\omega_{[-1,1]}(t)=\frac{1}{\pi \sqrt{1-t^{2}}}, \quad t \in(-1,1) .
$$

The following general fine zero spacing theorem was proved by B. Simon [29] and by the author [31] (recall that $\omega_{S}$ is the equilibrium density of the support $S$ of $\rho$ ).

Theorem 6.5 (Simon, Totik, 2008-2009) If $\rho \in \operatorname{Reg}$ and $w(t):=d \rho(t) / d t$ is continuous and positive at $a z_{0} \in \operatorname{Int}(S)$, then

$$
\lim _{n \rightarrow \infty} n \omega_{S}\left(z_{0}\right)\left(x_{j+1, n}-x_{j, n}\right)=1, \quad x_{j, n}-z_{0}=O(1 / n) .
$$


Furthermore, if $w>0$ is continuous on an interval $(a, b)$, then

$$
\lim _{n \rightarrow \infty} n \omega_{S}\left(x_{j}\right)\left(x_{j+1, n}-x_{j, n}\right)=1
$$

uniformly for $x_{j} \in[a+\varepsilon, b-\varepsilon]$.

It is quite remarkable that local spacing $x_{j+1}-x_{j}$ of zeros not only reflect (via $\left.\omega_{S}(x)\right)$ the (global) support of the measure, but also the position of the zero $x_{j}$ inside that support.

It is also true that if $\log w \in L^{1}(I)$ on some interval $I$, then (24) is true at almost all $z_{0} \in I$, see [31]. It is an open problem if (24) is true (say on $[-1,1]$ ) almost everywhere if, instead of $\log w \in L^{1}(I)$, we assume only the Erdős-Turán condition $w>0$ a.e.

\section{Erdős weights}

Besides orthogonal polynomials with respect to measures with compact support, orthogonal polynomials associated with weights on the whole real line have important applications. The prototypes are the Hermite polynomials associated with the weight function $w(x)=\exp \left(-x^{2}\right)$. If $d \rho(x)=w(x) d x$ is supported on the whole real line, then the zeros $z_{j, n}$ of the $n$-th orthogonal polynomials spread out: the largest zero $x_{n, n}$ tends to $\infty$ and the smallest zero $x_{1, n}$ tends to $-\infty$ as $n \rightarrow \infty$. So in this case one cannot speak of classical zero distribution. One rather considers so called contracted zeros that are obtained by transforming the interval $\left[x_{1, n}, x_{n, n}\right]$ linearly onto $[-1,1]$, and considering the zeros under this linear transformation. Note that this contraction brings all the zeros to $[-1,1]$, and if these contracted zeros have an asymptotic distribution $\sigma$, then $\sigma$ is called the contracted distribution of the zeros.

In the paper [8] Erdős proved the following.

Theorem 7.1 (Erdös, 1969) Let $0<w(x)<C$ on the real line, and assume that to every $\varepsilon>0$ there is an $x_{\varepsilon}$ such that for every $|x|>x_{\varepsilon}$ if $y$ is of the same sign as $x$ and $|y| \geq(1+\varepsilon)|x|$, then

$$
w(y)<w(x)^{2}
$$

holds. Then the contracted zero distribution of the corresponding orthogonal polynomials is the Chebyshev (arcsine) distribution.

It is easy to see that the condition (26) implies

$$
w(x)=o\left(e^{-|x|^{\alpha}}\right), \quad|x| \rightarrow \infty
$$

for all $\alpha$. In that same paper Erdös conjectured that (27) alone is sufficient for arcsine contracted zero distribution, but without further regularity this may not be true (a note by Lubinsky). However, under some regularity of the weight (like monotonicity around infinity) the results of [18, Theorem 14.2] and [20, 
Theorem 12.2] imply the conjecture (a note by Lubinsky), but those conditions are not as simple as (26).

Why do the conditions (26) and (27) appear in this respect? Already Erdős noticed that if $w(x)=\exp \left(-|x|^{\alpha}\right)$ with some $\alpha>0$, then the contracted zero distribution is not the Chebyshev distribution (since then it has been calculated that it is

$$
\left.\frac{\alpha}{\pi} \int_{|t|}^{1} \frac{u^{\alpha-1}}{\sqrt{u^{2}-t^{2}}} d u, \quad t \in[-1,1]\right),
$$

so one needs faster decrease to get arcsine distribution. Today weights satisfying (27) are called Erdős weights. The theory (orthogonal polynomials, approximation theory, polynomial inequalities) of Erdős weights has been developed by Lubinsky and Levin (and coauthors) in a series of papers and in the monographs [20] and [18]. There is an analogue on a finite interval: there those weights are called Erdős weights that vanish at the endpoints faster than any power of $x$; typical examples $\exp \left(-1 /\left(1-x^{2}\right)^{\alpha}\right), \exp \left(-\exp \left(1 /\left(1-x^{2}\right)^{\alpha}\right)\right)$.

\section{Critical points of polynomials}

Let $P_{n}$ be a polynomial of degree $n$, let $z_{1}, \ldots, z_{n}$ be its zeros and $\xi_{1}, \ldots, \xi_{n-1}$ the zeros of $P_{n}^{\prime}$.

The classical Gauss-Lucas theorem from the mid 1800's claims that every $\xi_{j}$ is in the convex hull of $\left\{z_{1}, \ldots, z_{n}\right\}$.

Erdős and I. Niven simultaneously with N. G. de Bruijn and T. A. Springer proved in 1947-48 that

$$
\frac{1}{n-1} \sum_{j=1}^{n-1}\left|\Im \xi_{j}\right| \leq \frac{1}{n} \sum_{k=1}^{n}\left|\Im z_{k}\right|,
$$

which implies (the reader is asked to do it!)

$$
\frac{1}{n-1} \sum_{j=1}^{n-1}\left|\xi_{j}\right| \leq \frac{1}{n} \sum_{k=1}^{n}\left|z_{k}\right| .
$$

This latter theorem lead to a fascinating area about the location of critical points $\xi_{j}$. First of all, it was extended by de Bruijn and Springer [4]: for all positive integer $m$

$$
\frac{1}{n-1} \sum_{j=1}^{n-1}\left|\xi_{j}\right|^{m} \leq \frac{1}{n} \sum_{k=1}^{n}\left|z_{k}\right|^{m} .
$$

They also conjectured that if $\varphi: \mathbf{C} \rightarrow \mathbf{R}_{+}$is convex (in the classical sense that $\varphi(\alpha z+(1-\alpha) w) \leq \alpha \varphi(z)+(1-\alpha) \varphi(w)$ for all $z, w$ and $0<\alpha<1)$, then

$$
\frac{1}{n-1} \sum_{j=1}^{n-1} \varphi\left(\xi_{j}\right) \leq \frac{1}{n} \sum_{k=1}^{n} \varphi\left(z_{k}\right) .
$$


Now this has known to be a very strong property through the works in the theory of majorization by Weyl, Birkhoff and Hardy-Littlewood-Pólya. This conjecture of de Bruijn and Springer remained open for more than half a century, and there were several related conjectures (see e.g. [23] and [25]) about the relationship between the zeros $\xi_{j}$ and $z_{k}$.

Many of these conjectures have been resolved by S. M. Malamud [23] and R. Pereira [25] in two simultaneous and independent papers in 2003. To state their theorem let us recall that an $(n-1) \times n$ size $\mathcal{A}=\left(a_{i j}\right)$ matrix is doubly stochastic if

- $a_{i j} \geq 0$

- each row-sum equals 1 , and

- each column-sum equals $(n-1) / n$.

Let

$$
\mathbf{Z}=\left(\begin{array}{c}
z_{1} \\
\vdots \\
z_{n}
\end{array}\right) \quad \boldsymbol{\Xi}=\left(\begin{array}{c}
\xi_{1} \\
\vdots \\
\xi_{n-1}
\end{array}\right)
$$

With these the key property is

Theorem 8.1 (Malamud, Pereira, 2003) There is a doubly stochastic matrix $\mathcal{A}$ such that $\Xi=\mathcal{A} \mathbf{Z}$.

The Gauss-Lucas theorem, the de Brjuin-Springer conjecture etc. are all immediate consequences. Indeed, we have

$$
\xi_{j}=\sum_{k=1}^{n} a_{j k} z_{k}
$$

so if $\varphi$ is convex then

$$
\begin{aligned}
& \frac{1}{n-1} \sum_{j=1}^{n-1} \varphi\left(\xi_{j}\right) \leq \frac{1}{n-1} \sum_{j=1}^{n-1} \sum_{k=1}^{n} a_{j k} \varphi\left(z_{k}\right) \\
& =\frac{1}{n-1} \sum_{k=1}^{n} \varphi\left(z_{k}\right) \sum_{j=1}^{n-1} a_{j k}=\frac{1}{n} \sum_{k=1}^{n} \varphi\left(z_{k}\right) .
\end{aligned}
$$

Other examples:

1)

$$
\frac{1}{n-1} \sum_{j=1}^{n-1}\left|\Re \xi_{j}\right|^{m} \leq \frac{1}{n} \sum_{k=1}^{n}\left|\Re z_{k}\right|^{m}, \quad m \geq 1 .
$$


2) If all zeros lie in the upper-half plane, then

$$
\left(\prod_{k=1}^{n} \Im z_{k}\right)^{1 / n} \leq\left(\prod_{j=1}^{n-1} \Im \xi_{j}\right)^{1 /(n-1)}
$$

Erdös would have loved these results particularly that their proof is quite simple. Malamud and Pereira developed related theories of matrix operations (inverse spectral theorems for normal matrices resp. differentiators), and they obtained Theorem 8.1 as a consequence. But if one only wants to prove Theorem 8.1, then the Malamud-Pereira argument is rather simple (we present Pereira's proof without differentiators). Indeed, we may assume $P_{n}$ to have leading coefficient 1. Let $\mathbf{E}_{1}, \ldots, \mathbf{E}_{n}$ be the standard orthonormal basis in $\mathbf{C}^{n}$, $\mathbf{A}$ the diagonal matrix/operator with diagonal entries $z_{1}, \ldots, z_{n}$, and let $\mathbf{v}=(1,1, \ldots, 1)^{T} / \sqrt{n}$. With this

$$
\mathbf{v}^{T}\left(x \mathbf{I}_{n}-\mathbf{A}\right)^{-1} \mathbf{v}=\frac{1}{n} \sum_{j=1}^{n}\left(x-z_{j}\right)^{-1}=\frac{1}{n} \frac{P_{n}^{\prime}(x)}{P_{n}(x)} .
$$

Let $\mathbf{e}_{n}=\mathbf{v}, \mathbf{e}_{n}^{\perp}$ its orthogonal complement and $\mathbf{P}$ the orthogonal projection onto $\mathbf{e}_{n}^{\perp}$. Choose an orthonormal basis $\mathbf{e}_{1}, \ldots, \mathbf{e}_{n-1}$ in $\mathbf{e}_{n}^{\perp}$ in which PA $\left.\right|_{\mathbf{e}_{n}^{\perp}}$ has a triangular matrix $\tilde{\mathbf{B}}$. Then $\mathbf{e}_{1}, \ldots, \mathbf{e}_{n}$ is an orthonormal basis in $\mathbf{C}^{n}$ and $\tilde{\mathbf{B}}$ is the $(n-1) \times(n-1)$ principal minor of the matrix $\tilde{\mathbf{A}}$ of the operator $\mathbf{A}$ in that basis. Now if $\tilde{\mathbf{v}}=(0, \ldots, 0,1)^{T}$ is the representation of $\mathbf{v}=\mathbf{e}_{n}$ in the basis $\mathbf{e}_{1}, \ldots, \mathbf{e}_{n}$, then

$$
\tilde{\mathbf{v}}^{T}\left(x \mathbf{I}_{n}-\tilde{\mathbf{A}}\right)^{-1} \tilde{\mathbf{v}}=\left(\left(x \mathbf{I}_{n}-\mathbf{A}\right)^{-1} \mathbf{e}_{n}, \mathbf{e}_{n}\right)=\mathbf{v}^{T}\left(x \mathbf{I}_{n}-\mathbf{A}\right)^{-1} \mathbf{v}=\frac{1}{n} \frac{P_{n}^{\prime}(x)}{P_{n}(x)}
$$

and

$$
\tilde{\mathbf{v}}^{T}\left(x \mathbf{I}_{n}-\tilde{\mathbf{A}}\right)^{-1} \tilde{\mathbf{v}}=\operatorname{det}\left(x \mathbf{I}_{n-1}-\tilde{\mathbf{B}}\right) / \operatorname{det}\left(x \mathbf{I}_{n}-\tilde{\mathbf{A}}\right)
$$

because both sides give the $(n, n)$ element of the matrix $\left(x \mathbf{I}_{n}-\tilde{\mathbf{A}}\right)^{-1}$. Since the denominator on the right is the characteristic polynomial of $\tilde{\mathbf{A}}$, which is the same as the characteristic polynomial of $\mathbf{A}$ i.e. $P_{n}(x)$, we get that $P_{n}^{\prime}(x) / n=$ $\operatorname{det}\left(x \mathbf{I}_{n-1}-\tilde{\mathbf{B}}\right)$. Therefore, the diagonal elements in $\tilde{\mathbf{B}}$ (the eigenvalues of $\tilde{\mathbf{B}}$ ) are $\xi_{1}, \ldots, \xi_{n-1}$, the zeros of $P_{n}^{\prime}$. With $\mathbf{e}_{j}=\sum_{k=1}^{n}\left(\mathbf{e}_{j}, \mathbf{E}_{k}\right) \mathbf{E}_{k}, \quad j=1, \ldots, n-1$, we have then for $1 \leq j \leq n-1, \tilde{\mathbf{e}}_{j}=(0, \ldots, 0,1,0, \ldots, 0)^{T}$ (with the 1 in the $j$-th position)

$$
\xi_{j}=\tilde{\mathbf{e}}_{j}^{T} \tilde{\mathbf{B}} \tilde{\mathbf{e}}_{j}=\tilde{\mathbf{e}}_{j}^{T} \tilde{\mathbf{A}} \tilde{\mathbf{e}}_{j}=\left(\mathbf{A} \mathbf{e}_{j}, \mathbf{e}_{j}\right)=\sum_{k=1}^{n} z_{k}\left|\left(\mathbf{e}_{j}, \mathbf{E}_{k}\right)\right|^{2} .
$$

Now this is the required representation, since $\sum_{k=1}^{n}\left|\left(\mathbf{e}_{j}, \mathbf{E}_{k}\right)\right|^{2}=\left\|\mathbf{e}_{j}\right\|^{2}=1$ and $\sum_{j=1}^{n-1}\left|\left(\mathbf{e}_{j}, \mathbf{E}_{k}\right)\right|^{2}=(n-1) / n$ because $\left|\left(\mathbf{e}_{n}, \mathbf{E}_{k}\right)\right|^{2}+\sum_{j=1}^{n-1}\left|\left(\mathbf{e}_{j}, \mathbf{E}_{k}\right)\right|^{2}=\left\|\mathbf{E}_{k}\right\|^{2}=$ 1 and $\left|\left(\mathbf{e}_{n}, \mathbf{E}_{k}\right)\right|^{2}=\left|\left(\mathbf{v}, \mathbf{E}_{k}\right)\right|^{2}=1 / n$. 
The author thanks L. Kérchy, D. S. Lubinsky and the referee for their valuable suggestions concerning the presentation.

\section{References}

[1] V. V. Andrievskii and H-P. Blatt, Discrepancy of signed measures and polynomial approximation, Springer Monographs in Mathematics. SpringerVerlag, New York, 2002.

[2] V. V. Andrievskii and H-P. Blatt, Erdős-Turán type theorems on quasiconformal curves and arcs, J. Approx. Theory, 97(1999), 334-365.

[3] P. Borwein, Paul Erdős and polynomials, Paul Erdős and his mathematics, I (Budapest, 1999), 161-174, Bolyai Soc. Math. Stud., 11, János Bolyai Math. Soc., Budapest, 2002.

[4] N. G. de Bruijn and T. A. Springer, On the zeros of a polynomial and of its derivative, II, Nederl. Akad. Wetensch., 50(1947) 264-270, = Indagationes Math., 9(1947), 458-464.

[5] T. Erdélyi, Markov-Bernstein type inequalities for polynomials under Erdős type constraints, Paul Erdös and his mathematics, I (Budapest, 1999), 219239, Bolyai Soc. Math. Stud., 11, János Bolyai Math. Soc., Budapest, 2002.

[6] T. Erdélyi, Polynomials with Littlewood-type coefficient constraints, Approximation theory, X (St. Louis, MO, 2001), 153-196, Innov. Appl. Math., Vanderbilt Univ. Press, Nashville, TN, 2002.

[7] P. Erdős, On the uniform distribution of the roots of certain polynomials, Ann. Math., 43(1942), 59-64.

[8] P. Erdős, On the distribution of the roots of orthogonal polynomials, Proceedings of the Conference on the Constructive Theory of Functions (Approximation Theory) (Budapest, 1969), pp. 145-150. Akadémiai Kiadó, Budapest, 1972.

[9] P. Erdős and I. Niven, On the roots of a polynomial and its derivative, Bull. Amer. Math. Soc., 54(1948), 184-190.

[10] P. Erdős and P. Turán, On interpolation I, Ann. Math., 38(1937), 142-155.

[11] P. Erdős and P. Turán, On interpolation II, On the distribution of the fundamental points of Lagrange interpolation, Ann. Math., 39(1938), 703724 .

[12] P. Erdős and P. Turán, On interpolation III, Interpolatory theory of polynomials, Ann. Math., 41(1940), 510-553.

[13] P. Erdős and P. Turán, On the distribution of certain sequences of points, Ann. Math., 41(1940), 162-173. 
[14] P. Erdős and P. Turán, On a problem in the theory of uniform distribution, I and II, Indag. Math., 10(1948), 370-378, 406-413.

[15] P. Erdős and P. Turán, On the distribution of roots of polynomials, Ann. Math., 51(1950), 105-119.

[16] A. E. Eremenko and W. Hayman, On the length of lemniscates, Paul Erdös and his mathematics, I (Budapest, 1999), 241-242, Bolyai Soc. Math. Stud., 11, János Bolyai Math. Soc., Budapest, 2002.

[17] T. Ganelius, Sequences of analytic functions and their zeros, Ark. Mat., 3(1953), 1-50.

[18] E. Levin and D. S. Lubinsky, Orthogonal polynomials for exponential weights, CMS Books in Mathematics/Ouvrages de Mathmatiques de la SMC, 4. Springer-Verlag, New York, 2001.

[19] A. L. Levin, and D. S. Lubinsky, Applications of universality limits to zeros and reproducing kernels of orthogonal polynomials, J. Approx. Theory., 150(2008), 69-95.

[20] D. S. Lubinsky, Strong asymptotics for extremal errors and polynomials associated with Erdös-type weights, Pitman Research Notes in Mathematics Series, 202. Longman Scientific \& Technical, Harlow; copublished in the United States with John Wiley \& Sons, Inc., New York, 1989.

[21] D. S. Lubinsky, A new approach to universality limits involving orthogonal polynomials, Ann. of Math., 170(2009), 915-939.

[22] D. S. Lubinsky, A taste of Erdős on interpolation, Paul Erdös and his mathematics, I (Budapest, 1999), 423-454, Bolyai Soc. Math. Stud., 11, János Bolyai Math. Soc., Budapest, 2002.

[23] S. M. Malamud, Inverse spectral problem for normal matrices and the Gauss-Lucas theorem, Trans. Amer. Math. Soc., 357(2005), 4043-4064.

[24] G. Mastroianni and V. Totik, Uniform spacing of zeros of orthogonal polynomials, Constr. Approx., 32(2010), 181-192.

[25] R. Pereira, Differentiators and the geometry of polynomials, J. Math. Anal. Appl., 285(2003), 336-348.

[26] E. A. Rakhmanov, On the asymptotics of the ratio of orthogonal polynomials, Math. USSR Sb., 32 (1977), 199-213.

[27] E. A. Rakhmanov, The asymptotic behavior of the ratio of orthogonal polynomials. II. (Russian) Mat. Sb. (N.S.) 118(160)(1982), 104-117.

[28] T. Ransford, Potential Theory in the Complex plane, Cambridge University Press, Cambridge, 1995 
[29] B. Simon, Two extensions of Lubinsky's universality theorem, J. D'Analyse Math., 105(2008), 345-362.

[30] H. Stahl and V. Totik, General Orthogonal Polynomials Encyclopedia of Mathematics, 43, Cambridge University Press, New York 1992

[31] V. Totik, Universality and fine zero spacing on general sets, Arkiv för Math., 47(2009), 361-391.

[32] V. Totik and with T. Varga, Nonsymmetric fast decreasing polynomials and applications, J. Math. Anal. Appl., 394(2012), 378-390.

[33] T. Varga, Uniform spacing of zeros of orthogonal polynomials for locally doubling measures, Analysis, (to appear).

[34] P. Vértesi, On the Lebesgue function and Lebesgue constant: A tribute to Paul Erdős, Paul Erdős and his mathematics, I (Budapest, 1999), 705-728, Bolyai Soc. Math. Stud., 11, János Bolyai Math. Soc., Budapest, 2002.

[35] P. Vértesi, Paul Erdős and interpolation: problems, results and new developments, (this volume)

Bolyai Institute

Analysis Research Group of the Hungarian Academy os Sciences

University of Szeged

Szeged

Aradi v. tere 1, 6720, Hungary

and

Department of Mathematics and Statistics

University of South Florida

4202 E. Fowler Ave, CMC342

Tampa, FL 33620-5700, USA

totik@mail.usf.edu 